\title{
Vomiting as harbinger for Graves' disease
}

\section{Bejinariu E, Banerjee R, Soo SC. Diabetes and Endocrinology Department}

Luton and Dunstable University Hospital

Introduction: Isolated vomiting is a rare often forgotten presentation of Graves's disease ${ }^{1}$ and can lead to delayed diagnosis. Multiple case reports quote vomiting in thyrotoxicosis co-presenting with Addisonian crisis ${ }^{2}$, diabetic ketoacidosis ${ }^{3,4}$ or with abnormal liver function and jaundice 5,6 . Another common association is hyperemesis gravidarum. Vomiting in paraneoplastic hyperthyroidism occurs through a similar mechanism of beta-HCG secretion, mainly related to germ-line tumours ${ }^{7}$. We present a case of severe prolonged vomiting caused by Graves' disease.

Case presentation: A 46 years old postmenopausal woman of Afro-Caribbean origin presented with unremitting vomiting and dramatic 38 Kilograms weight loss over 2-3 months. She reported tiredness, headaches and anxiety. Her heart rate was $91 / \mathrm{min}$ sinus rhythm, BP $115 / 80$, temperature $37.2^{\circ} \mathrm{C}$. She had no palpable thyroid nor thyroid eye signs. Thyroid function tests showed suppressed TSH $<0.01 \mathrm{mU} / \mathrm{L}$ and elevated fT4 86.9 $\mathrm{pmol} / \mathrm{L}$. The Burch and Wartofsky's score was 20 (temperature 37.2 , heart rate 91 in sinus rhythm, absent agitation or CNS symptoms), indicating this was not a thyroid storm presentation.

Further investigations: Thyroid uptake scan and anti-thyroid antibodies confirmed Graves's disease. Liver function tests were normal. Thyrotoxicosis related hypercalcaemia was noted (corrected calcium $2.75 \mathrm{mmol} / \mathrm{l}$ with low parathyroid hormone 1.3 $\mathrm{pmol} / \mathrm{l})$. Coeliac, autoimmune screen and beta-HCG were negative. Gastroscopy with duodenal biopsy and CT body imaging were normal.

\begin{tabular}{|l|l|l|l|l|l|l|l|}
\hline & $\begin{array}{l}\text { Normal } \\
\text { range }\end{array}$ & $\mathbf{1 5 . 0 3 . 1 6}$ & $\mathbf{2 1 . 0 3 . 1 6}$ & $\mathbf{2 2 . 0 3 . 1 6}$ & $\mathbf{0 4 . 0 5 . 1 6}$ & $\mathbf{1 1 . 0 7 . 1 6}$ & $\mathbf{2 9 . 0 9 . 1 6}$ \\
\hline TSH (mU/) & $0.27-4.20$ & $<0.01$ & $<0.01$ & $<0.01$ & 0.02 & $<0.01$ & $<0.01$ \\
\hline FT4 (pmo//) & $11.0-22.0$ & 85.9 & 50.5 & 35.2 & 18.8 & 25.6 & 37.3 \\
\hline FT3 (pmo//) & $3.7-6.8$ & & & & 9.0 & 13.2 & \\
\hline $\begin{array}{l}\text { PTU dose } \\
\text { (24 hours) }\end{array}$ & & $300 \mathrm{mg}$ & $300 \mathrm{mg}$ & $300 \mathrm{mg}$ & $200 \mathrm{mg}$ & $300 \mathrm{mg}$ & $400 \mathrm{mg}$ \\
\hline TRAB (U/) & $<1.4$ & & & & & 1.4 & \\
\hline TPO (U/ml) & $<34$ & & 234 & & 186 & & \\
\hline cCa (mmol/) $)$ & & 2.75 & & 2.47 & 2.51 & 2.68 & \\
\hline $\begin{array}{l}\text { PTH } \\
\text { (pmol/l) }\end{array}$ & $1.6-6.9$ & 1.3 & & & 5.3 & & \\
\hline
\end{tabular}

Progress: The patient required a prolonged two weeks admission for managing her unremitting vomiting during which Propylthiouracil and Propranolol were administered via nasogastric tube. Vomiting was refractory to antiemetics but improved alongside amelioration of hyperthyroidism with antithyroid medication.

Conclusion: Vomiting is commonly associated with endocrine disorders. Literature review reports female predominance of thyrotoxic vomiting. Previous case reports of isolated vomiting have no Graves's thyroid eye signs making early diagnosis challenging. Typically, they were associated with significant weight loss in the context of a delayed diagnosis, and persistent tachycardia as diagnostic clues. Characteristically such vomiting fails to respond to usual antiemetics, but responds to antithyroid medications. Glucocorticoids are also useful adjuncts in bringing symptoms under control ${ }^{11}$.

\begin{tabular}{|l|l|}
\hline \multicolumn{2}{|l|}{ Mechanisms of thyrotoxic vomiting } \\
\hline $\begin{array}{l}\text { Increased beta-adrenergic } \\
\text { activity }\end{array}$ & $\begin{array}{l}\text { Increases the number of beta-adrenergic } \\
\text { receptors }^{8,9}\end{array}$ \\
\hline \multirow{2}{*}{ Increased thyroid hormones } & Stimulates the chemical trigger zone \\
\cline { 2 - 2 } & $\begin{array}{l}\text { Decreases gastric emptying and causes pyloric } \\
\text { sphincter malfunction }\end{array}$ \\
\cline { 2 - 2 } & $\begin{array}{l}\text { Increases magnesium urinary excretion. } \\
\text { Hypomagnesaemia inhibits gastric smooth } \\
\text { muscle }\end{array}$ \\
\hline Increased oestrogen level in \\
patients of both sexes
\end{tabular}

Recommendations: Increased awareness of vomiting as presentation of thyrotoxicosis is necessary in order to avoid a delayed diagnosis and over-investigations. There is a potential risk of precipitating a thyroid storm while exposing patients unnecessarily to invasive investigations such as endoscopy or exploratory laparoscopy.

Ruling out alternative aetiologies is also important. Rare paraneoplastic thyrotoxic conditions may present as such.

\section{Luton and Dunstable University Hospital}

Follow up: There was recurrence of isolated vomiting with weight loss 6 months following presentation during Propylthiouracil titration. Her Propylthiouracil dose has currently been titrated back to $400 \mathrm{mg}$ daily.

\begin{tabular}{|c|c|c|c|c|c|}
\hline & sc & $\begin{array}{l}\text { Burch and Wartofsky's Diag } \\
>45 \text { is highly suggestive; } 2\end{array}$ & stic & $\begin{array}{l}\text { iteria for thyroid storm }{ }^{19} \\
\text { uggestive and }<25 \text { is improbable }\end{array}$ & \\
\hline Temperat & & Central nervous system & & GI system & \\
\hline $37.2-37.7$ & 5 & Mild agitation & 10 & Moderate (diarrhoea, nausea/vomiting, & 10 \\
\hline $37.8-38.2$ & 10 & Moderate (delirium, psychosis, & 10 & abdominal pain) & \\
\hline $38.3-38.8$ & 15 & extreme lethargy) & & Severe (unexplained jaundice) & \\
\hline 38.9-39.4 & 20 & Severe (seizures, coma) & & & 20 \\
\hline 39.4-39.9 & 25 & & 30 & & \\
\hline$>40.0$ & 30 & & & & \\
\hline Triggerin & & Cardiovascular system & & & \\
\hline & 0 & $\begin{array}{l}\text { Heart rate } \\
\text { 99-109 }\end{array}$ & 5 & $\begin{array}{l}\text { Atrial fibrillation } \\
\text { Heart Failure }\end{array}$ & 10 \\
\hline Positive & 10 & $110-119$ & 10 & Mild(peripheral oedema) & 5 \\
\hline & & $120-129$ & 15 & Moderate (bibasilar rales) & 10 \\
\hline & & $130-139$ & 20 & Severe (pulmonary oedema) & 15 \\
\hline & & $>=140$ & 25 & & \\
\hline
\end{tabular}

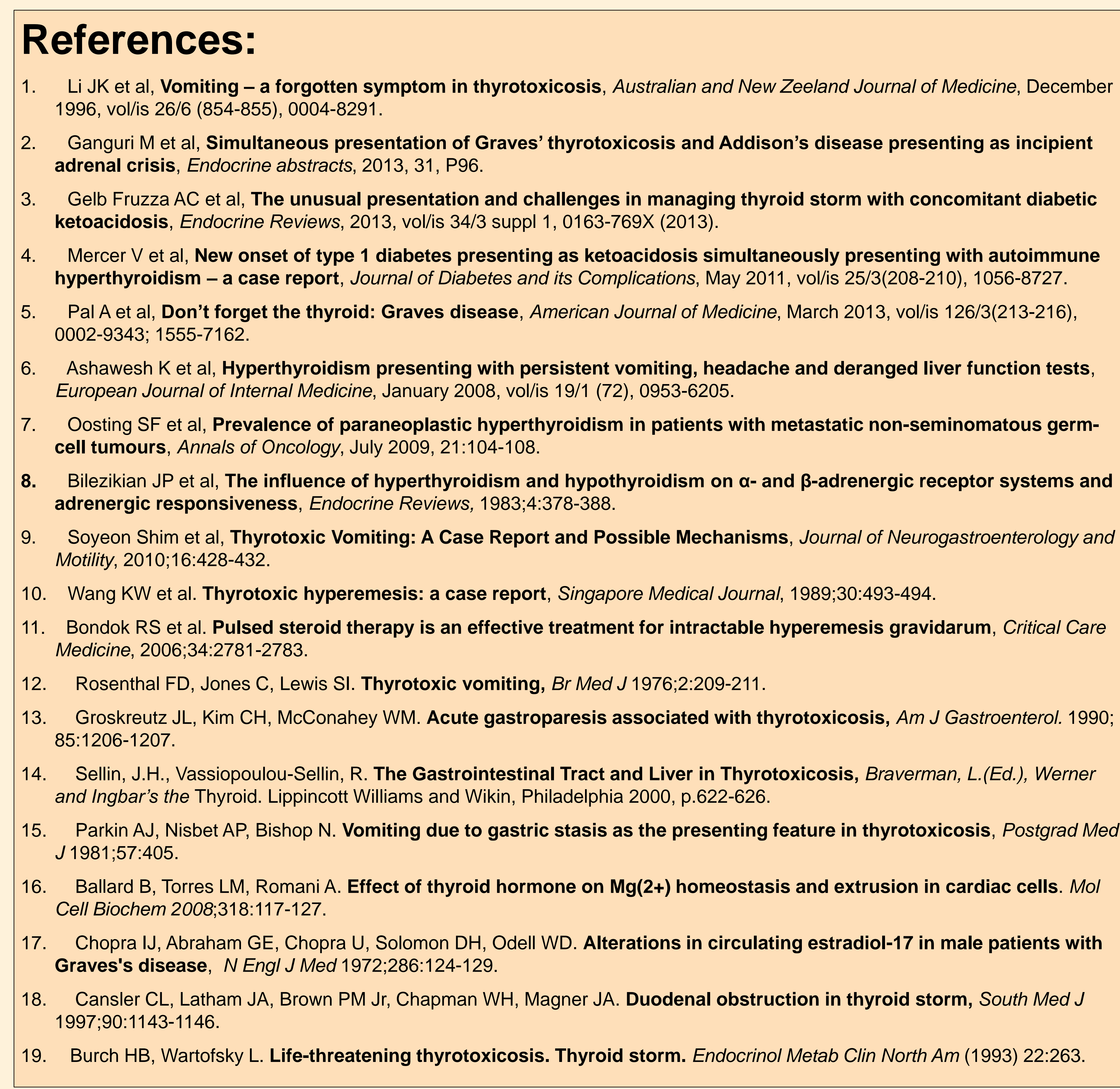

